This manuscript contains material that is original and not previously published in text or on the Internet, nor is it being considered elsewhere until a decision is made as to its acceptability by the Journal of Strength and Conditioning Research Editorial Review Board.

\title{
Concurrent training followed by detraining: does the resistance training intensity
}

matter?

\section{Type of article: Original Investigation}

Running Head: Strength training intensity during concurrent training

António C. Sousa ${ }^{1,2}$; Daniel A. Marinho ${ }^{1,2}$; Maria H. Gil ${ }^{1,2}$; Mikel Izquierdo ${ }^{3,4}$; David Rodríguez-Rosell $^{5}$; Henrique P. Neiva ${ }^{1,2}$; Mário C. Marques ${ }^{1,2}$

${ }^{1}$ University of Beira Interior. Department of Sport Sciences, UBI, Covilhã, Portugal;

${ }^{2}$ Research Center in Sport Sciences, Health Sciences and Human Development, CIDESD, Portugal;

${ }^{3}$ Department of Health Sciences, Public University of Navarre, Navarre, Spain.

${ }^{4}$ GICAEDS Group, Faculty of Physical Culture, Sport and Recreation, University of Santo

Tomás, Bogotá, D.C, Colombia

${ }^{5}$ Research Center on Physical and Athletic Performance, Pablo de Olavide University, Seville.

\section{Corresponding Author and address:}

Mikel Izquierdo, PhD

Department of Health Sciences, Public University of Navarra (Navarra)

Campus of Tudela, Av. de Tarazona s/n. 31500 Tudela (Navarra) SPAIN

Tel + 34948 417876; email: mikel.izquierdo@gmail.com

The authors disclose funding received for this work from any of the following organizations: National Institutes of Health (NIH); Welcome Trust; Howard Hughes Medical Institute (HHMI); and other(s). 


\section{ABSTRACT}

The aim of the present study was to analyze the training and detraining effects of concurrent aerobic training and resistance training against three different external loads on strength and aerobic variables. Thirty-two men were randomly assigned to four groups: low-load (LLG, n=9), moderate-load (MLG, n=9), high-load (HLG, n=8), and control group (CG, $n=6)$. Resistance training consisted of FS with a low-load (40-55\% 1RM), a moderate-load $(55-70 \%$ 1RM) or a high-load $(70-85 \%$ 1RM) combined with jump and sprint exercises. Aerobic training was performed at $75 \%$ of the maximal aerobic speed for 15-20 min. The training period lasted for 8-weeks, followed by 4-weeks detraining. Pre, post-training and post-detraining evaluations included $20 \mathrm{~m}$ running sprints $(0-10 \mathrm{~m}$ : T10; 0 20m: T20), shuttle run test, countermovement vertical jump test (CMJ), and loading test $(1 \mathrm{RM})$ in full-squat $(\mathrm{FS})$. All the experimental groups showed improvements $(\mathrm{p}<0.05)$ in all the parameters assessed, except the LLG for T10 and the HLG for T20. The LLG, MLG and HLG showed great changes in $1 \mathrm{RM}$ and $\mathrm{VO}_{2} \max$ compared with the $\mathrm{CG}(\mathrm{p}<0.05)$, whereas the HLG and MLG showed a greater percentage change than the CG in T10 $(\mathrm{p}<0.001)$ and CMJ $(\mathrm{p}<0.05)$. The 4-week detraining period resulted in detrimental effects in all variables analyzed for all three experimental groups. In conclusion, our results suggest that strength training programs with low, moderate, or high external loads combined with low-intensity aerobic training could be effective for producing significant gains in strength and aerobic capacities. Moreover, the higher loads used increased gains in explosive efforts.

KEYWORDS: Endurance training, weight training, load-magnitude, sprint performance, jump performance, full squat training 


\section{INTRODUCTION}

Concurrent training (CT) has become a contemporary topic for coaches, strength and conditioning professionals and researchers because a large number of sports require both strength and aerobic capacities for maximize performance $(30,41,51)$. However, resistance and endurance trainings produce divergent metabolic and morphological adaptations with little overlap between them $(12,41)$. Therefore, it seems necessary to find optimal combinations of both types of training regimes to obtain maximum simultaneous development of strength and endurance capacities.

Studies analyzing the neuromuscular adaptations and performance improvements associated with CT have reported inconsistent results. While concurrent training does not alter the ability to positively adapt to endurance training $(6,51)$, most studies have indicated that CT regimens appear to inhibit strength, hypertrophy and power development compared with resistance training alone $(15,18,27)$. Nevertheless, some experiments have reported little or no negative effect on strength gains with the addition of aerobic training $(1,35,48)$.

In addition to large influence of the interindividual variation in response to a training program $(25,32)$, the effects of CT on strength gains may vary markedly due to a large number of design factors, including the mode, frequency, duration, type of exercises, volume and intensity used during both resistance and aerobic training, different sequences and recovery times between resistance and aerobic training sessions, training history of participants, and dependent variables selected $(13,29,51)$. The effect of most of these variables has already received considerable attention in previous studies and reviews $(41,51)$. However, to the best of our knowledge, a question that remains ignored in the literature is the possibility of manipulating the load magnitude during resistance training. 
In addition, most of resistance exercises used in studies analyzing the effect of CT on physical performance $(3,7,15,18,23,25,27)$ were open-chain, isolated, isotonic or machinebased exercises (i.e. leg extension and flexion, seated hamstring curl, leg curl, leg press, isometric plantar flexion, calf rise). It appears that resistance training programs which preferably include open-chain exercises may not provide adequate movement pattern specificity for optimal performance improvements in closed-chain sporting movements such as running (2). Therefore, it has been indicated that future investigations should include traditional multi-joint resistance exercises because are believed to be superior for eliciting optimal neuromuscular adaptations and increasing the force capabilities of the leg musculature (2). Since (i) the training load seems to be the most important variable to consider when designing a resistance training program (11), and (ii) the exercises selected in a resistance training programme can influence the magnitude of neuromuscular adaptations (2), gains in strength and endurance variables during CT may be directly influenced by the load magnitude and exercise used during resistance training. Thus, the first aim of the present study was to analyze the effect of three CT programs that only differed in the load magnitude used during the full squat training on performance in vertical jumping, sprint, leg strength and endurance capacity.

Additionally, interruptions in training sessions due to several factors are normal in any sport $(26,38,40,42)$. For this reason, knowing the effects of a detraining period (DT) could be important for designing better training strategies. The detraining adaptations following strength or endurance training alone have been widely studied in different populations $(8,9,26,31,36)$ Unfortunately, the effect of training cessation after CT has received less scientific attention $(4,45,46,49)$. Moreover, although abrupt cessation of intense physical training is associated with a decline of physical performance (38-40), detraininginduced changes in performance after concurrent training are linked with multiple factors 
$(16,31,36,46,49,50)$ among which is included the relative intensity used during previous resistance program. Therefore, the second aim of the present study was to analyze the effects of 4-week DT following concurrent training programs differing in load magnitude used during resistance training on different strength and aerobic parameters.

\section{METHODS}

\section{Experimental Approach to the Problem}

An experimental research design was used to compare the effects of three concurrent resistance and aerobic training programs only differing in load magnitude used during resistance training $(40-55 \% 1 \mathrm{RM}$ vs. $55-70 \% 1 \mathrm{RM}$ vs. $70-85 \%$ 1RM) on physical performance, and the subsequence detraining adaptations. To address this, thirty-six male physically active men were randomly assigned to control group $(\mathrm{CG})$ or resistance training group with low loads (LLG), moderate loads (MLG) or high load (HLG). The players assigned to experimental groups performed resistance training combined with endurance, while players assigned to CG merely undertook daily life activities. All the experimental groups trained twice a week for 8 weeks using a CT regimen. All subjects were evaluated using a battery of tests performed in two sessions separated by a $48 \mathrm{~h}$ rest interval. During the first testing session, the participants performed the $20 \mathrm{~m}$ running sprints and the $20 \mathrm{~m}$ shuttle run test. During the second testing session, subjects executed the countermovement vertical jump test (CMJ), and an isoinertial loading test in full squat exercise. During the 2 weeks preceding this study, four preliminary familiarization sessions were undertaken to ensure a proper execution technique in both full squat and CMJ exercises. To evaluate the DT effects, the strength and aerobic parameters were tested after four weeks of training cessation. Throughout this period, the participants were asked refrain from participating in 
regular exercise programs aimed at developing or maintaining strength and aerobic capacity.

\section{Subjects}

Thirty-six male physically active men volunteered to participate in this study. After an initial evaluation, the participants were matched according to their estimated one-repetition maximum $\left(1 \mathrm{RM}_{\mathrm{est}}\right)$ in full-squat exercises $(\mathrm{FS})$ and then randomly assigned to four groups depending on the loading magnitude used during resistance training, as follows: i) a lowload group (LLG, 40-55\% 1RM), a moderate-load group (MLG, 55-70\% 1RM), a highload group (HLG, 70-85\% 1RM), and a control group (CG). Due to injury or illness, four participant (one from the HLG and three from the CG) were absent from the post-testing sessions. Thus, of the 36 initially enrolled participants, only 32 successfully completed the entire study. Player characteristics are displayed in Table 1. Participants in the CG were asked not to perform any type of resistance or aerobic training during the experimental period. All the participants provided written informed consent to the experimental procedures after the possible benefits and risks of participation were explained to them. The investigation was conducted in accordance with the Declaration of Helsinki and was approved by the local Research Ethics Committee.

$$
\text { ***Please insert Table } 1 \text { near here*** }
$$

\section{Procedures}

Neuromuscular performance was assessed before (Pre), after the 8-week training period (Post 1), and after the 4-week detraining period (Post 2) using a battery of tests performed in two sessions separated by a $48 \mathrm{~h}$ rest interval. Testing sessions were performed at the same time of day for each participant under the same environmental conditions $\left(\sim 20^{\circ} \mathrm{C}\right.$ and 
$\sim 60 \%$ humidity). Body mass and height (Seca Instruments, Ltd., Hamburg, Germany) were measured prior to the warm-up protocol in the first testing session. Strong verbal encouragement was provided during all tests to motivate participants to give a maximal effort.

Running sprints: Each participant performed three $20 \mathrm{~m}$ sprints separated by a $3 \mathrm{~min}$ rest. Photocell timing gates (Brower photocells, Wireless Sprint System, USA) were placed at 0, 10 and $20 \mathrm{~m}$ so that the times needed to cover $0-10 \mathrm{~m}$ (T10) and $0-20 \mathrm{~m}$ (T20) could be determined. A standing start with the lead-off foot placed $1 \mathrm{~m}$ behind the first timing gate was used. The average of the best two sprints was used for the analysis. Warm-up consisted of 5 minutes of running at a self-selected intensity, 5 minutes of joint mobilization exercises, followed by several sets of progressively faster 30-m running accelerations. Reliability for T20 as measured by the coefficient of variation (CV) was $3.8 \%$, while the intra-class correlation coefficient (ICC) was 0.94 .

Shuttle run test: The $20 \mathrm{~m}$ multistage shuttle run test was administered according to the original version described by Léger (28). The initial running velocity was set at $8.5 \mathrm{~km} \cdot \mathrm{h}^{-1}$ and was gradually increased in $0.5 \mathrm{~km} \cdot \mathrm{h}^{-1}$ each minute (14). The test was terminated when a participant failed to reach the appropriate marker in the allotted time twice or could no longer maintain the pace. The number of laps completed was recorded. Estimated maximum oxygen consumption $\left(\mathrm{VO}_{2 \mathrm{max}}, \mathrm{ml} \cdot \mathrm{kg}^{-1} \cdot \mathrm{min}^{-1}\right)$ was calculated based on the maximal speed (MAS) reached before participants were unable to keep up with the audio recording, as follows: $-27.4+6 \cdot \operatorname{MAS}(28)$. 
Vertical jump test: The jump height was determined using a contact mat connected to an electronic power timer, control box and handset (Globus Ergojump, Italy). Each participant performed three maximal CMJs with their hands on their hips, separated by $1 \mathrm{~min}$ rests. The highest value was recorded for the subsequent analysis. The ICC was 0.96 , and the CV was $3.2 \%$.

Isoinertial squat loading test: A Smith machine (Multipower Fitness Line, Peroga, Murcia, Spain) was used for this test. A detailed description of the testing procedures used in this study was recently reported elsewhere (14). The initial load was set at $17 \mathrm{~kg}$ and progressively increased in $10 \mathrm{~kg}$ increments until the attained mean propulsive velocity (MPV) was $\sim 1.00 \mathrm{~m} \cdot \mathrm{s}^{-1}$ (range $0.95-1.05 \mathrm{~m} \cdot \mathrm{s}^{-1}$ ) (14). The participants performed 3 repetitions with each load, with $3 \mathrm{~min}$ recovery. A linear velocity transducer (T-Force System, Ergotech, Murcia, Spain) was used to register bar velocity. The $1 \mathrm{RM}_{\mathrm{est}}$ was calculated based on the MPV attained against the heaviest load lifted, as follows: (100 . load $) /\left(-5.961 \cdot \mathrm{MPV}^{2}\right)-(50.71 \cdot \mathrm{MPV})+117(44)$.

\section{Training program}

The descriptive characteristics of the training programs completed by each group are presented in Table 2. The resistance training session comprised full squat, vertical jump and sprint exercises. Approximately 2-3min rest periods were allowed between each set and exercise. The participants were instructed to perform all exercises at maximal intended velocity to obtain the highest possible gains (43). The loads used by each participant in the full squat exercise were assigned according to $1 \mathrm{RM}$ est obtained in the initial isoinertial squat loading test. Thus, the relative intensity of the full squat exercise progressively increased from $40 \%$ to $55 \% 1 \mathrm{RM}, 55 \%$ to $70 \% 1 \mathrm{RM}$, and $70 \%$ to $85 \%$ 1RM for LLG, MLG and HLG, respectively. Because strength was expected to increase with training, an 
intermediate isoinertial squat loading test was carried out after 4 weeks of training in order to perform the necessary load adjustments for each training group. Aerobic training was performed $20 \mathrm{~min}$ after the participants completed the resistance training. All the experimental groups completed the same aerobic training regimen, which consisted of 1520 min performing the $20 \mathrm{~m}$ shuttle run exercise at $75 \%$ of the maximal individual speed reached during the $20 \mathrm{~m}$ multistage shuttle run test. As for strength training, participants were assessed in the $20 \mathrm{~m}$ shuttle run test after 4 weeks of training in order to perform the necessary adjustments for each training group. At least 2 trained researchers supervised each workout session and recorded the compliance and individual workout data during each training session. All participants were instructed to maintain their normal daily activities throughout the study. The participants did not undertake any additional strength or aerobic training activities during the testing, training, and detraining periods.

\section{Statistical analysis}

The values of each variable are presented as mean \pm standard deviation (SD). Homogeneity of variance across groups (LLG vs. MLG vs. HLG vs. CG) was verified using the Levene test, whereas the normality of distribution of the data was examined with the Shapiro-Wilk test. A 4 (group: LLG, MLG, HLG, CG) x 3 (time: Pre, Post 1, Post 2) repeated measures analysis of variances (ANOVA) was calculated for each variable. Sphericity was checked using Mauchly's test. Percentage of change for each variable was calculated [(post pre/pre) $\times 100$ ] and a one-way ANOVA was conducted to examine between-group differences with tukey post-hoc comparisons (LLG vs. MLG vs. HLG vs. CG) to clarify the interaction. In addition to this null hypothesis testing, the data were assessed for clinical significance using an approach based on the magnitudes of change (20). The effect 
sizes (ES) were calculated using Cohen's $d$ (9) to estimate the magnitude of the training effect on the selected neuromuscular variables within each group. The threshold values for assessing the magnitudes of the standardized effects were $0.20,0.60,1.20$ and 2.00 for small, moderate, large and very large magnitudes, respectively. Probabilities were also calculated to establish whether the true (unknown) differences were lower than, similar to, or higher than the smallest worthwhile difference or change (0.2 multiplied by the between-subject SD) (20). The quantitative chances of obtaining higher or lower differences were evaluated as follows: $1 \%$, almost certainly not; $1-5 \%$, very unlikely; $5-$ 25\%, unlikely; 25-75\%, possible; 75-95\%, likely; 95-99\%, very likely; 99\%, almost certain. If the chances of having higher or lower values than the smallest worthwhile difference were both $>5 \%$, the true difference was assessed as unclear. Inferential statistics based on the interpretation of the magnitude of effects were calculated using a purposebuilt spreadsheet for the analysis of controlled trials (19). The statistical analyses were performed using SPSS software version 18.0 (SPSS, Inc., Chicago, IL, USA). Statistical significance was established at the $\mathrm{p} \leq 0.05$ level.

\title{
RESULTS
}

Data for all variables analyzed were homogeneous and normally distributed ( $\mathrm{p}>0.05)$. There were no significant differences between groups at baseline for any analyzed variable. The mean values, percentage of change and intra-group ES for all variables analyzed during Pre, Post 1 and Post 2 are reported in Table 3 (LLG), Table 4 (MLG), and Table 5 (HLG).

\author{
***Please insert Table 3 near here*** \\ ***Please insert Table 4 near here*** \\ ***Please insert Table 5 near here***
}


All the experimental groups showed improvements $(\mathrm{p}<0.05-0.001)$ in all the variables assessed except the LLG in T10 and the HLG in T20 (Tables 3, 4 and 5). No changes took place in the CG. The magnitude of change for LLG was from small (T10, T20, 1RM $\mathrm{est}_{\text {and }}$ VO2max) to moderate (CMJ). For MLG, the standardized effects were small (T10, T20 and $\left.1 \mathrm{RM}_{\mathrm{est}}\right)$ and moderate (CMJ and $\left.\mathrm{VO} 2 \mathrm{max}\right)$, whereas for $\mathrm{HLG}$, the qualitative outcome relative to ES was small (T20 and $\left.1 \mathrm{RM}_{\mathrm{est}}\right)$, moderate (T10 and VO2max) or large (CMJ), depending to the assessed variable.

After the training period, significant "time $\times$ group" interactions were observed for T10 ( $p$ $<0.001), \mathrm{CMJ}(\mathrm{p}<0.01), 1 \mathrm{RM}_{\mathrm{est}}(\mathrm{p}<0.01)$ and VO2max $(\mathrm{p}<0.001)$, whereas there was no "time $\times$ group" interaction in T20 ( $\mathrm{p}=0.349)$. The one-way ANOVA indicated that all the experimental groups showed significantly greater percent changes from Pre to Post 1 for $1 \mathrm{RM}_{\mathrm{est}}(\mathrm{p}<0.05-0.01)$ and $\mathrm{VO} 2 \max (\mathrm{p}<0.05-0.05)$ compared to CG, whereas the HLG and MLG also showed greater percentage of change than CG in T10 (p < 0.001) and CMJ ( $<$ < 0.05), respectively (Table 6; Figure 1).

The 4-week DT period produced an important detriment effect on all the variables analyzed for all the experimental groups. Most of these variables returned to initial values or lower after the rest period (Tables 3, 4 and 5). In fact, no differences were found between Pre and Post 2 in any studied variable for any experimental group. In addition, no significant differences were found between the three-trained groups and the CG at Post 2 for any variable.

$* * *$ Please insert Table 6 near here***

***Please insert Figure 1 near here $* * *$ 


\section{DISCUSSION}

To the best of our knowledge, this was the first study focused on analyzing the training and detraining effects after concurrent training programs differing in the relative intensity $(\% 1 \mathrm{RM})$ used during resistance training regime on strength and aerobic performance in physical active men. The main finding of the present study was that the all three experimental groups showed significant and practical improvements in different performance variables including jump, running sprint, maximal strength and $\mathrm{VO}_{2} \mathrm{max}$. Thus, it appears that resistance training programs consisting in full squat exercise with low (40 - 55\% 1RM), moderate (55 - 70\% 1RM), or high (70 - 85\% 1RM) loads combined with the same low-intensity aerobic training $\left(75 \% \mathrm{VO}_{2} \max \right)$ could be equally effective for producing significant gains in strength and endurance capacities. In addition, the DT period resulted in significant performance decrements in all variables assessed for all experimental groups. These results could be of great interest for coaches and strength and conditioning professionals to optimize training programs in those sports modalities to require combinations of both components of strength and endurance training for maximize performance.

\section{Strength performance}

All three experimental groups showed significant $(\mathrm{p}<0.05-0.01)$ improvements in $1 \mathrm{RM}_{\mathrm{est}}$ after training period. However, changes reported in LLG (13.9\%; ES: 0.57), MLG (9.9\%; ES: 0.40) and HLG (11.4\%; ES: 0.47) were lower than those reported in previous studies $(\sim 20 \%)(15,23,35)$ and meta-analyses (ES:1.30) (51) that assessed the effects of CT on strength development in untrained male participants. Although have been described that continuous aerobic training would be predicted to have minimal interference on strength gains using either high load or moderate load resistance training protocols $(6,13)$, it is 
possible that the short rest period between resistance and aerobic training in the present study ( 20 min) may have induced a greater degree of interference than previous studies (51). Thus, our results confirmed the need to separate resistance and aerobic training sessions to optimize strength gains (13). In addition, the use of only one resistance exercise (full squat) has also been able to influence in the lower gains found in the present study compared to other studies $(7,15,23,35)$ in which several resistance exercises were used (e.g., knee flexion and extension, leg curl, leg press, calf raise). Comparison between experimental groups showed no significant differences in strength gains between LLG, MLG and HLG. However, the analysis of practical inferences resulted in a possible better effect on $1 \mathrm{RM}_{\mathrm{est}}$ for LLG compared to MLG and HLG. These results are in agreement with previous studies indicating that resistance training programs that include training with moderate to high loads and repetitions at or near the point of muscle failure lead to lower strength gains compared with the use of a moderate number of repetitions for not training to repetition failure $(6,13,22,37)$.

\section{Sprint and Vertical Jump Performance}

Only few studies $(5,21,34)$ have analyzed the effect of CT on jump performance, while, to the best of our knowledge, no studies have examined the influence of CT on running sprints in adult individuals. In the present study, all three combinations resulted in lowmoderate improvements in CMJ (11.6 - 13.9\%; ES: 0.61 - 1.27) and sprint times (1.0 3.5\%; ES: 0.20 - 0.63). These improvements in CMJ were greater than previous studies conducted with untrained individuals $(9.0$ - 3.3\%). Thus, although aerobic training (continuous and high-intensity run training) has been reported to cause deterioration in the capacity of the neuromuscular system to rapidly generate force (17), it appears that adding explosive exercises (jumps and accelerations) along with the full-squat exercise executing 
each repetition at maximal intended velocity could attenuate the interference on adaptations to short and high intensity efforts.

Regarding the load magnitude used during resistance training, the present study showed no significant differences for Pre-Post changes in jump and sprint variables between experimental groups. However, there was a slight trend toward greater intra-group ES for HLG compared with LLG and MLG in T10 and CMJ. In addition, HLG showed a likely greater effect than LLG and MLG in T10, while practically worthwhile difference was possibly more beneficial in favour of MLG compared to LLG. For the rest of comparisons, the differences between LLG, MLG and HLG were unclear. These results appear to be in contrast with a recent meta-analysis (47) which indicated that high-load resistance training alone resulted in lesser sprint ES $(\mathrm{ES}=0.52)$ compared with lower loads $(\mathrm{ES}=0.97)$. However, our results seem to indicate that, when resistance training is combined with continuous aerobic training, using moderate to high loads is more effective for improving jump and sprint performance than those with low loads.

\section{Aerobic Performance}

The training period resulted in similar improvements in $\mathrm{VO}_{2} \mathrm{max}$ for all three experimental groups. These changes $(\sim 12-15 \%)$ were comparable to those reported in previous studies ( 7 - 18\%) performing CT or aerobic regimens alone $(3,15,18,21,34)$. Therefore, although the present study did not include a group that underwent aerobic training alone, our results appear to be in line with previous reports, suggesting that CT does not affect the development of $\mathrm{VO}_{2} \max$ in untrained or resistance-trained individuals. In addition, as a remarkable contribution of the present study, our results suggest that load magnitude used during resistance training do not effect on changes in aerobic performance, as no significant differences were found in $\mathrm{VO}_{2}$ max gains between LLG, MLG and HLG. 


\section{Detraining Effect}

The DT period resulted in a marked and similar reduction in physical performance for all three experimental groups, with a partial $\left(\mathrm{CMJ}\right.$ and $\left.1 \mathrm{RM}_{\mathrm{est}}\right)$ or complete $(\mathrm{T} 10, \mathrm{~T} 20$ and $\mathrm{VO}_{2} \mathrm{max}$ ) reversals of the adaptations obtained during 8-week training period. This is in accordance with previous studies that have shown important $\mathrm{VO}_{2}$ max declines (4-14\%) with short-term training cessation in trained and untrained individuals (38). However, studies conducted with elementary school students using CT have shown both significant loss (45) and no changes (46) in this variable. In relation to sprint performance, several studies using a CT training period $(36,45,46)$ have shown that the sprint time in 10,20 and $30 \mathrm{~m}$ remained unchanged or only decreases slightly during the DT period. Discrepancies with our results could be due to differences in the age of the participants and the training program configurations $(36,45,46)$.

According to several studies and review analyzing the effect of detraining period after CT training or resistance training alone $(26,38)$, the loss of maximal strength $(4-7 \%)$ and CMJ height (5 - 9\%) in the present study were lower compared aerobic performance. Since CMJ performance depends largely on the maximal strength of the leg extensors $(10,52)$, it is possible that the lower reduction in $\mathrm{CMJ}$ performance was associated with the maintenance of $1 \mathrm{RM}_{\mathrm{est}}$. In accordance with our results, other studies have shown no significant changes in CMJ performance after 4-6 weeks of cessation of resistance training $(24,26,33)$. However, it appears that when resistance training is combined with aerobic training, both maximal strength and $\mathrm{CMJ}$ height trend to descend to a greater extent after DT period $(45,46)$. 
The present study has some limitations need to be addressed. Obviously, one of the main limitations of the present study is the low number of subjects in each group. Thus, some effects are associated with large confidence limits for the intra- and between-group change differences. Therefore, we can not be sure whether differences within and between groups would have been clearer with a greater number of subjects in each experimental group. In addition, the present study evaluated the efficacy of aerobic training and a specific resistance training regimen consisting in full squat exercise alone. It is possible that the use of only one resistance exercise may have been a limitation for strength gains during CT. In addition, this type of resistance training has also been able to influence the degree of loss of physical performance during the DT period. However, since the main aim of the present study was to analyze the training and detraining effects of combined resistance training programs against three different external loads with the same aerobic training on strength and aerobic variables, we consider it appropriate not to include additional resistance exercises to avoid increasing the number of confounding factors such as number of exercises, rest time between exercises, type of exercises (e.g., multi-join vs. isolated, closed- vs. open-chain, isoinertial vs. isotonic), or fatigue accumulated. However, a comparison of the relative efficacy of different resistance training regimens combined with different aerobic training seems to be an interesting topic for future research. Finally, we should acknowledge that different participants, for instance, experienced ones could lead to other results and further investigation should also be developed in this regard.

\section{CONCLUSIONS}

In brief, the results of the present study indicated that 8-weeks of resistance training programs with different loads combined with low-intensity aerobic training improved strength and aerobic capacities, regardless of training intensity used during resistance training. Despite the similar improvements, resistance trainings with loads higher than 55\% 
of $1 \mathrm{RM}_{\mathrm{est}}$ are suggested to increase changes in explosive efforts, such as short runs (T10 $\mathrm{m})$ and CMJ. In addition, 4-weeks of DT compromised previous gains, mainly in $\mathrm{VO}_{2} \mathrm{max}$ and sprint time variables.

\section{PRACTICAL APPLICATIONS}

The results seems to suggest that performing strength training with low, moderate, or high external loads combined with low-intensity aerobic training regimen is beneficial for strength and aerobic development in healthy adult men. Furthermore, choosing higher loads during strength training can lead to increased gains in explosive efforts. Despite our data highlight that 8 -weeks of training are sufficient to verify enhancements, it takes only 4-weeks without training to return to the initial values. This should be considered when designing concurrent training in sports clubs to improve its efficiency. Thereupon, this experiment provides a new path in order to integrate both strength and aerobic regimens in the same session.

\section{ACKNOWLEDGMENTS}

This project was supported by the National Funds through FCT - Portuguese Foundation for Science and Technology (UID/DTP/04045/2013) - and the European Fund for Regional Development (FEDER) allocated by European Union through the COMPETE 2020 Programme (POCI-01-0145-FEDER-006969) - competitiveness and internationalization (POCI). 


\section{REFERENCES}

1. Balabinis, CP, Psarakis, CH, Moukas, M, Vassiliou, MP, and Behrakis, PK. Early phase changes by concurrent endurance and strength training. J Strength Cond Res 17: 393-401, 2003.

2. Beattie, K, Kenny, IC, Lyons, M, and Carson, BP. The effect of strength training on performance in endurance athletes. Sports Med 44: 845-865, 2014.

3. Bell, GJ, Petersen, SR, Wessel, J, Bagnall, K, and Quinney, HA. Adaptations to endurance and low velocity resistance training performed in a sequence. Can J Sport Sci 16: 186-192, 1991.

4. Carvalho, MJ, Marques, E, and Mota, J. Training and detraining effects on functional fitness after a multicomponent training in older women. Gerontology 55: 41-48, 2009.

5. Chtara, M, Chaouachi, A, Levin, GT, Chaouachi, M, Chamari, K, Amri, M, and Laursen, PB. Effect of concurrent endurance and circuit resistance training sequence on muscular strength and power development. J Strength Cond Res 22: 1037-1045, 2008.

6. Docherty, D, and Sporer, B. A proposed model for examining the interference phenomenon between concurrent aerobic and strength training. Sports Med 30: 385-394, 2000 .

7. Dolezal, BA, and Potteiger, JA. Concurrent resistance and endurance training influence basal metabolic rate in nondieting individuals. J Appl Physiol (1985) 85: 695700, 1998.

8. Eastwood, A, Bourdon, PC, Snowden, KR, and Gore, CJ. Detraining decreases $\mathrm{Hb}$ (mass) of triathletes. Int J Sports Med 33: 253-257, 2012.

9. Faigenbaum, AD, Westcott, WL, Micheli, LJ, Outerbridge, AR, Long, CJ, LarosaLoud, R, and Zaichkowskt, LD. The effects of strength training and detraining on children. J Strength Cond Res 10: 109-114, 1996.

10. Franco-Marquez, F, Rodriguez-Rosell, D, Gonzalez-Suarez, JM, Pareja-Blanco, F, Mora-Custodio, R, Yanez-Garcia, JM, and Gonzalez-Badillo, JJ. Effects of Combined Resistance Training and Plyometrics on Physical Performance in Young Soccer Players. Int J Sports Med 36: 906-914, 2015.

11. Fry, AC. The role of resistance exercise intensity on muscle fibre adaptations. Sports Med 34: 663-679, 2004.

12. Fyfe, JJ, Bishop, DJ, and Stepto, NK. Interference between concurrent resistance and endurance exercise: molecular bases and the role of individual training variables. Sports Med 44: 743-762, 2014.

13. Garcia-Pallares, J, and Izquierdo, M. Strategies to optimize concurrent training of strength and aerobic fitness for rowing and canoeing. Sports Med 41: 329-343, 2011.

14. Gonzalez-Badillo, JJ, Pareja-Blanco, F, Rodriguez-Rosell, D, Abad-Herencia, JL, Del Ojo-Lopez, JJ, and Sanchez-Medina, L. Effects of velocity-based resistance training on young soccer players of different ages. J Strength Cond Res 29: 1329-1338, 2015.

15. Hakkinen, K, Alen, M, Kraemer, WJ, Gorostiaga, E, Izquierdo, M, Rusko, H, Mikkola, J, Hakkinen, A, Valkeinen, H, Kaarakainen, E, Romu, S, Erola, V, Ahtiainen, J, and Paavolainen, L. Neuromuscular adaptations during concurrent strength and endurance training versus strength training. Eur J Appl Physiol 89: 42-52, 2003.

16. Hasegawa, Y, Ijichi, T, Kurosawa, Y, Hamaoka, T, and Goto, K. Planned Overreaching and Subsequent Short-term Detraining Enhance Cycle Sprint Performance. Int J Sports Med 36: 666-671, 2015.

17. Hennessy, LC, and Watson, AWS. The interference effects of training for strength and endurance simultaneously. J Strength Cond Res 8: 12-19, 1994. 
18. Hickson, RC. Interference of strength development by simultaneously training for strength and endurance. Eur J Appl Physiol Occup Physiol 45: 255-263, 1980.

19. Hopkins, WG. Analysis of a pre-post controlled trial (Excel spreadsheet). newstats.org/xParallelGroupTrial.xls Retrieved 10 February, 2012, from http://www.sportsci.org. 2006.

20. Hopkins, WG, Marshall, SW, Batterham, AM, and Hanin, J. Progressive statistics for studies in sports medicine and exercise science. Med Sci Sports Exerc 41: 3-13, 2009.

21. Hunter, G, Demment, R, and Miller, D. Development of strength and maximum oxygen uptake during simultaneous training for strength and endurance. J Sports Med Phys Fitness 27: 269-275, 1987.

22. Izquierdo-Gabarren, M, Gonzalez De Txabarri Exposito, R, Garcia-pallares, J, Sanchez-medina, L, De Villarreal, ES, and Izquierdo, M. Concurrent endurance and strength training not to failure optimizes performance gains. Med Sci Sports Exerc 42: 1191-1199, 2010.

23. Izquierdo, M, Hakkinen, K, Ibanez, J, Kraemer, WJ, and Gorostiaga, EM. Effects of combined resistance and cardiovascular training on strength, power, muscle crosssectional area, and endurance markers in middle-aged men. Eur J Appl Physiol 94: 70-75, 2005.

24. Kannas, TM, Amiridis, IG, Arabatzi, F, Katis, A, and Kellis, E. Changes in specific jumping performance after detraining period. J Sports Med Phys Fitness 55: 1150-1156, 2015.

25. Karavirta, L, Hakkinen, K, Kauhanen, A, Arija-Blazquez, A, Sillanpaa, E, Rinkinen, N, and Hakkinen, A. Individual responses to combined endurance and strength training in older adults. Med Sci Sports Exerc 43: 484-490, 2011.

26. Kraemer, WJ, Koziris, LP, Ratamess, NA, Hakkinen, K, NT, TR-M, Fry, AC, Gordon, SE, Volek, JS, French, DN, Rubin, MR, Gomez, AL, Sharman, MJ, Michael Lynch, J, Izquierdo, M, Newton, RU, and Fleck, SJ. Detraining produces minimal changes in physical performance and hormonal variables in recreationally strength-trained men. $J$ Strength Cond Res 16: 373-382, 2002.

27. Kraemer, WJ, Patton, JF, Gordon, SE, Harman, EA, Deschenes, MR, Reynolds, K, Newton, RU, Triplett, NT, and Dziados, JE. Compatibility of high-intensity strength and endurance training on hormonal and skeletal muscle adaptations. J Appl Physiol 78: 976989, 1995.

28. Leger, LA, Mercier, D, Gadoury, C, and Lambert, J. The multistage 20 metre shuttle run test for aerobic fitness. J Sports Sci 6: 93-101, 1988.

29. Leveritt, M, Abernethy, PJ, Barry, B, and Logan, PA. Concurrent strength and endurance training: the influence of dependent variable selection. J Strength Cond Res 17: 503-508, 2003.

30. Leveritt, M, Abernethy, PJ, Barry, BK, and Logan, PA. Concurrent strength and endurance training. A review. Sports Med 28: 413-427, 1999.

31. Lo, MS, Lin, LL, Yao, WJ, and Ma, MC. Training and detraining effects of the resistance vs. endurance program on body composition, body size, and physical performance in young men. J Strength Cond Res 25: 2246-2254, 2011.

32. Mann, TN, Lamberts, RP, and Lambert, MI. High responders and low responders: factors associated with individual variation in response to standardized training. Sports Med 44: 1113-1124, 2014.

33. Marques, MC, and Gonzalez-Badillo, JJ. In-season resistance training and detraining in professional team handball players. J Strength Cond Res 20: 563-571, 2006.

34. McCarthy, JP, Agre, JC, Graf, BK, Pozniak, MA, and Vailas, AC. Compatibility of adaptive responses with combining strength and endurance training. Med Sci Sports Exerc 27: 429-436, 1995. 
35. McCarthy, JP, Pozniak, MA, and Agre, JC. Neuromuscular adaptations to concurrent strength and endurance training. Med Sci Sports Exerc 34: 511-519, 2002.

36. Meylan, CM, Cronin, JB, Oliver, JL, Hopkins, WG, and Contreras, B. The effect of maturation on adaptations to strength training and detraining in 11-15-year-olds. Scand $J$ Med Sci Sports 24: e156-164, 2014.

37. Mora-Custodio, R, Rodríguez-Rosell, D, Pareja-Blanco, F, Yánez-García, JM, and González-Badillo, JJ. Effect of low-vs. moderate-load squat training on strength, jump and sprint performance in physically active women. Int J Sports Med 37: 476-482, 2016.

38. Mujika, I, and Padilla, S. Detraining: loss of training-induced physiological and performance adaptations. Part I: short term insufficient training stimulus. Sports Med 30: 79-87, 2000.

39. Mujika, I, and Padilla, S. Cardiorespiratory and metabolic characteristics of detraining in humans. Med Sci Sports Exerc 33: 413-421, 2001.

40. Mujika, I, and Padilla, S. Muscular characteristics of detraining in humans. Med Sci Sports Exerc 33: 1297-1303, 2001.

41. Nader, GA. Concurrent strength and endurance training: from molecules to man. Med Sci Sports Exerc 38: 1965-1970, 2006.

42. Ormsbee, MJ, and Arciero, PJ. Detraining increases body fat and weight and decreases VO2peak and metabolic rate. J Strength Cond Res 26: 2087-2095, 2012.

43. Pareja-Blanco, F, Rodriguez-Rosell, D, Sanchez-Medina, L, Gorostiaga, EM, and Gonzalez-Badillo, JJ. Effect of movement velocity during resistance training on neuromuscular performance. Int J Sports Med 35: 916-924, 2014.

44. Sánchez-Medina, L, Pallarés, JG, Pérez, CE, Morán-Navarro, R, and GonzálezBadillo, JJ. Estimation of relative load from bar velocity in the full back squat exercise. Sports Med Int Open 1: E80-E88, 2017.

45. Santos, A, Marinho, DA, Costa, AM, Izquierdo, M, and Marques, MC. The effects of concurrent resistance and endurance training follow a specific detraining cycle in young school girls. J Hum Kinet 29A: 93-103, 2011.

46. Santos, AP, Marinho, DA, Costa, AM, Izquierdo, M, and Marques, MC. The effects of concurrent resistance and endurance training follow a detraining period in elementary school students. J Strength Cond Res 26: 1708-1716, 2012.

47. Seitz, LB, Reyes, A, Tran, TT, Saez de Villarreal, E, and Haff, GG. Increases in lower-body strength transfer positively to sprint performance: a systematic review with meta-analysis. Sports Med 44: 1693-1702, 2014.

48. Sillanpaa, E, Hakkinen, A, Nyman, K, Mattila, M, Cheng, S, Karavirta, L, Laaksonen, DE, Huuhka, N, Kraemer, WJ, and Hakkinen, K. Body composition and fitness during strength and/or endurance training in older men. Med Sci Sports Exerc 40: 950-958, 2008.

49. Toraman, NF. Short term and long term detraining: is there any difference between young-old and old people? Br J Sports Med 39: 561-564, 2005.

50. Toraman, NF, and Ayceman, N. Effects of six weeks of detraining on retention of functional fitness of old people after nine weeks of multicomponent training. $\mathrm{Br} J$ Sports Med 39: 565-568; discussion 568, 2005.

51. Wilson, JM, Marin, PJ, Rhea, MR, Wilson, SM, Loenneke, JP, and Anderson, JC. Concurrent training: a meta-analysis examining interference of aerobic and resistance exercises. J Strength Cond Res 26: 2293-2307, 2012.

52. Wisloff, U, Castagna, C, Helgerud, J, Jones, R, and Hoff, J. Strong correlation of maximal squat strength with sprint performance and vertical jump height in elite soccer players. Br J Sports Med 38: 285-288, 2004. 


\section{FIGURE LEGENDS}

Figure 1. Relative changes in performance variables (A: T10; B: T20; C: CMJ; D: $1 \mathrm{RM}_{\mathrm{est}}$; E: VO2max) from baseline in the low-load (LLG), moderate-load (MLG), high-load (HLG) and control group. Error bars represent $90 \%$ of confidence interval of changes from baseline to post-training and baseline to detraining. Statistically significant differences respect to $\mathrm{CG}: * \mathrm{p}<0.05, * * \mathrm{p}<0.01, * * * \mathrm{p}<0.001$. 
Table 1. Subject characteristics.

\begin{tabular}{lcccc} 
& \multicolumn{4}{c}{ Group } \\
\cline { 2 - 5 } \multicolumn{1}{c}{ Variable } & $\begin{array}{c}\text { LLG } \\
(n=8)\end{array}$ & $\begin{array}{c}\text { MLG } \\
(n=9)\end{array}$ & $\begin{array}{c}\text { HLG } \\
(n=9)\end{array}$ & $\begin{array}{c}\text { CG } \\
(n=6)\end{array}$ \\
\hline Age (years) & $20.6 \pm 0.9$ & $20.6 \pm 1.6$ & $20.6 \pm 1.9$ & $20.7 \pm 2.3$ \\
Height (m) & $1.80 \pm 0.1$ & $1.80 \pm 0.0$ & $1.80 \pm 0.1$ & $1.80 \pm 0.1$ \\
Body Mass (Kg) & $71.8 \pm 8.3$ & $68.5 \pm 10.4$ & $67.8 \pm 4.6$ & $70.1 \pm 4.8$ \\
\hline
\end{tabular}

Values are mean \pm SD.

LLG: Low-load group; MLG: Moderate-load group; HLG: High-load group; CG: Control group 
Table 2. Characteristics of the training program performed by the LLG, MLG and HLG groups.

\begin{tabular}{|c|c|c|c|c|c|c|c|c|c|c|c|c|c|c|c|}
\hline \multirow[b]{2}{*}{ Exercise } & \multicolumn{15}{|c|}{ Sessions } \\
\hline & 1 & 2 & 3 & 4 & 5 & 6 & 7 & 8 & 9 & 10 & 11 & 12 & 13 & 14 & 15 \\
\hline \multicolumn{16}{|c|}{ Full Squat (\% 1RM: SxR) } \\
\hline$L L G$ & $40: 3 \times 8$ & $40: 3 \times 8$ & $40: 3 \times 8$ & $45: 3 \times 8$ & $45: 3 \times 8$ & $45: 3 \times 8$ & $50: 3 \times 6$ & $3 \times 6$ & & $55: 3 \times 6$ & $55: 3 \times 6$ & $55: 3 \times 6$ & $50: 3 \times 6$ & $50: 3 \times 6$ & $40: 3 \times 6$ \\
\hline$M L G$ & $55: 3 \times 8$ & $55: 3 \times 8$ & $55: 3 \times 8$ & $60: 3 \times 6$ & $60: 3 \times 6$ & $60: 3 \times 6$ & $65: 3 \times 6$ & 6 & & $0: 3 \times 6$ & $70: 3 \times 6$ & $70: 3 \times 6$ & $65: 3 \times 6$ & $65: 3 \times 6$ & $60: 3 \times 6$ \\
\hline$H L G$ & $70: 3 \times 8$ & $70: 3 \times 8$ & $70: 3 \times 8$ & $75: 3 \times 8$ & $75: 3 \times 8$ & $75: 3 \times 6$ & $80: 3 \times 5$ & 5 & & $85: 3 \times 5$ & $85: 3 \times 5$ & $85: 3 \times 5$ & $80: 3 \times 5$ & $80: 3 \times 5$ & $75: 3 \times 8$ \\
\hline CMJ (SxR) & $2 \times 5$ & $2 \times 5$ & $2 \times 5$ & $2 \times 5$ & $2 \times 5$ & $2 \times 5$ & $2 \times 5$ & & $3 \times 5$ & $3 \times 5$ & $3 \times 5$ & $3 \times 5$ & $3 \times 5$ & $3 \times 5$ & $2 \times 5$ \\
\hline Sprint (SxD) & $2 \times 30 \mathrm{~m}$ & $2 \times 30 \mathrm{~m}$ & $2 \times 30 \mathrm{~m}$ & $3 \times 30 \mathrm{~m}$ & $3 \times 30 \mathrm{~m}$ & $3 \times 30 \mathrm{~m}$ & $3 \times 20 \mathrm{~m}$ & $3 \times 20 m$ & $3 \times 20 \mathrm{~m}$ & $4 \times 20 m$ & $4 \times 20 m$ & $4 \times 20 m$ & $3 \times 20 \mathrm{~m}$ & $3 \times 20 \mathrm{~m}$ & $2 \times 20 \mathrm{~m}$ \\
\hline $\begin{array}{l}\text { 20m Shuttle Run } \\
\text { (T x \%MAS) }\end{array}$ & $\begin{array}{l}15 \min x \\
75 \%\end{array}$ & $\begin{array}{l}15 \min \\
\times 75 \%\end{array}$ & $\begin{array}{l}15 \min \\
x 75 \%\end{array}$ & $\begin{array}{l}15 \min \\
x 75 \%\end{array}$ & $\begin{array}{l}20 \min \\
\times 75 \%\end{array}$ & $\begin{array}{l}20 \min \\
\times 75 \%\end{array}$ & $\begin{array}{l}20 \min \\
x 75 \%\end{array}$ & $\begin{array}{l}20 \min \\
x 75 \%\end{array}$ & $\begin{array}{l}15 \min \\
x 75 \%\end{array}$ & $\begin{array}{l}15 \min \\
\times 75 \%\end{array}$ & $\begin{array}{l}15 \min \\
\times 75 \%\end{array}$ & $\begin{array}{l}15 \min \\
\times 75 \%\end{array}$ & $\begin{array}{l}20 \min \\
\times 75 \%\end{array}$ & $\begin{array}{l}20 \min \\
x 75 \%\end{array}$ & $\begin{array}{l}20 \min \\
\times 75 \%\end{array}$ \\
\hline
\end{tabular}

LLG: Low-load group; MLG: Moderate-load group; HLG: High-load group; 1RM: One-repetition maximum; SxR: sets x repetitions; SxD: Sets x distance; Tx\%MAS: Time (min) x percentage of the maximal speed reached for each participant during the $20 \mathrm{~m}$ multistage shuttle run test. 
Table 3. Changes in selected neuromuscular performance variables from pre-training to post-training and detraining period for LLG.

\begin{tabular}{|c|c|c|c|c|c|c|c|c|c|c|c|c|}
\hline \multirow{2}{*}{ Variable } & \multirow[b]{2}{*}{ Pre } & \multirow[b]{2}{*}{ Post 1} & \multirow[b]{2}{*}{ Post 2} & \multicolumn{3}{|c|}{ Post 1 vs. Post 2} & \multicolumn{3}{|c|}{ Post 1 vs. Post 3} & \multicolumn{3}{|c|}{ Post 2 vs. Post 3} \\
\hline & & & & $p$-value & $\Delta( \pm 90 \% \mathrm{CI})$ & $\mathrm{ES}( \pm 90 \% \mathrm{CI})$ & $p$-value & $\Delta( \pm 90 \% \mathrm{CI})$ & $\mathrm{ES}( \pm 90 \% \mathrm{CI})$ & $p$-value & $\Delta( \pm 90 \% \mathrm{CI})$ & $\mathrm{ES}( \pm 90 \% \mathrm{CI})$ \\
\hline T10 (s) & $1.87 \pm 0.11$ & $1.84 \pm 0.09$ & $1.90 \pm 0.10$ & 0.148 & $-1.3 \pm 1.0$ & $0.20 \pm 0.15$ & 0.573 & $1.6 \pm 2.0$ & $-0.24 \pm 0.31$ & 0.129 & $2.9 \pm 2.3$ & $-0.44 \pm 0.34$ \\
\hline $\mathrm{T} 20(\mathrm{~s})$ & $3.21 \pm 0.15$ & $3.16 \pm 0.16$ & $3.22 \pm 0.15$ & 0.007 & $-1.5 \pm 0.6$ & $0.29 \pm 0.12$ & 1.000 & $0.5 \pm 0.6$ & $-0.09 \pm 0.18$ & 0.042 & $2.0 \pm 1.2$ & $-0.38 \pm 0.22$ \\
\hline $\mathrm{CMJ}(\mathrm{cm})$ & $33.8 \pm 5.1$ & $37.7 \pm 5.3$ & $34.2 \pm 5.1$ & 0.002 & $11.6 \pm 3.9$ & $0.61 \pm 0.19$ & 1.000 & $1.3 \pm 6.6$ & $0.07 \pm 0.36$ & 0.077 & $-9.3 \pm 6.4$ & $-0.54 \pm 0.33$ \\
\hline $1 \mathrm{RM}_{\mathrm{est}}(\mathrm{kg})$ & $81.9 \pm 17.0$ & $92.4 \pm 18.5$ & $85.5 \pm 16.0$ & 0.004 & $13.9 \pm 5.6$ & $0.57 \pm 0.22$ & 0.058 & & $0.25 \pm 0.16$ & 0.018 & $-7.1 \pm 3.4$ & $-0.33 \pm 0.16$ \\
\hline $\mathrm{VO}_{2} \max \left(\mathrm{ml} \cdot \mathrm{kg}^{-1} \cdot \mathrm{min}^{-1}\right)$ & $41.0 \pm 8.5$ & $46.7 \pm 7.2$ & $42.2 \pm 5.0$ & 0.000 & $15.2 \pm 5.0$ & $0.56 \pm 0.17$ & 1.000 & $4.3 \pm 9.6$ & $0.17 \pm 0.36$ & 0.089 & $-9.5 \pm 5.7$ & $-0.40 \pm 0.25$ \\
\hline
\end{tabular}

Data are mean \pm SD

LLG: Low-load group; Pre: initial evaluation; Post 1: Evaluation after training period; Post 2: evaluation after detraining period; $\Delta$ : percentage of change; ES: intragroup effect size; CI: confidence interval; T10: 10-m sprint time; T20: 20-m sprint time; $\mathrm{CMJ}$ : countermovement jump; $1 \mathrm{RM}_{\mathrm{est}}$ : estimated one-repetition maximum; $\mathrm{VO}_{2} \mathrm{max}$ estimated maximal oxygen uptake. 
Table 4. Changes in selected neuromuscular performance variables from pre-training to post-training and detraining period for MLG.

\begin{tabular}{|c|c|c|c|c|c|c|c|c|c|c|c|}
\hline \multirow[b]{2}{*}{ Variable } & \multirow[b]{2}{*}{ Pre } & \multirow[b]{2}{*}{ Post 1} & \multirow[b]{2}{*}{ Post 2} & \multicolumn{3}{|c|}{ Post 1 vs. Post 2} & \multicolumn{2}{|c|}{ Post 1 vs. Post 3} & \multicolumn{3}{|c|}{ Post 2 vs. Post 3} \\
\hline & & & & $p$-value & $\Delta( \pm 90 \% \mathrm{CI})$ & $\mathrm{ES}( \pm 90 \% \mathrm{CI})$ & $p$-value $\Delta( \pm 90 \% \mathrm{CI})$ & $\mathrm{ES}( \pm 90 \% \mathrm{CI})$ & $p$-value & $\Delta( \pm 90 \% \mathrm{CI})$ & $\mathrm{ES}( \pm 90 \% \mathrm{CI})$ \\
\hline T10 (s) & $1.83 \pm 0.06$ & $1.81 \pm 0.07$ & $1.87 \pm 0.09$ & 0.034 & $-1.0 \pm 0.6$ & $0.27 \pm 0.15$ & $2.0 \pm 1.7$ & $-0.51 \pm 0.47$ & 0.015 & $1.8 \pm 0.9$ & $-0.77 \pm 0.33$ \\
\hline $\mathrm{T} 20(\mathrm{~s})$ & $3.12 \pm 0.12$ & $3.05 \pm 0.13$ & $3.17 \pm 0.11$ & 0.003 & $-2.3 \pm 0.8$ & $0.56 \pm 0.20$ & $1.6 \pm 1.4$ & $-0.37 \pm 0.33$ & 0.009 & $4.0 \pm 1.8$ & $-0.92 \pm 0.41$ \\
\hline $\mathrm{CMJ}(\mathrm{cm})$ & $34.2 \pm 4.9$ & $38.8 \pm 3.8$ & $36.6 \pm 5.0$ & 0.005 & $13.9 \pm 6.4$ & $0.85 \pm 0.37$ & $7.2 \pm 6.6$ & $0.46 \pm 0.40$ & 0.115 & $-5.9 \pm 4.1$ & $-0.40 \pm 0.29$ \\
\hline $1 \mathrm{RM}_{\mathrm{est}}(\mathrm{kg})$ & $84.2 \pm 16.7$ & $92.6 \pm 18.6$ & $89.5 \pm 16.9$ & 0.022 & $9.9 \pm 4.7$ & $0.40 \pm 0.18$ & 0.16 & $0.23 \pm 0.19$ & 0.267 & $-4.0 \pm 3.8$ & $-0.17 \pm 0.17$ \\
\hline $\mathrm{VO}_{2} \max \left(\mathrm{ml} \cdot \mathrm{kg}^{-1} \cdot \mathrm{min}^{-1}\right)$ & $43.9 \pm 6.8$ & $49.0 \pm 5.7$ & $41.9 \pm 6.7$ & 0.001 & $12.1 \pm 4.7$ & $0.64 \pm 0.23$ & $-4.7 \pm 5.4$ & $-0.27 \pm 0.31$ & 0.001 & $-15.0 \pm 4.8$ & $-0.91 \pm 0.31$ \\
\hline
\end{tabular}

Data are mean \pm SD

MLG: Moderate-load group; Pre: initial evaluation; Post 1: Evaluation after training period; Post 2: evaluation after detraining period; $\Delta$ : percentage of change; ES: intragroup effect size; CI: confidence interval; T10: 10-m sprint time; T20: 20-m sprint time; CMJ: countermovement jump; 1 RM ${ }_{\mathrm{est}}$ : estimated one-repetition maximum; $\mathrm{VO}_{2}$ max: estimated maximal oxygen uptake. 
Table 5. Changes in selected neuromuscular performance variables from pre-training to post-training and detraining period for HLG.

\begin{tabular}{|c|c|c|c|c|c|c|c|c|c|c|c|c|}
\hline \multirow[b]{2}{*}{ Variable } & \multirow[b]{2}{*}{ Pre } & \multirow[b]{2}{*}{ Post 1} & \multirow[b]{2}{*}{ Post 2} & \multicolumn{3}{|c|}{ Post 1 vs. Post 2} & \multicolumn{3}{|c|}{ Post 1 vs. Post 3} & \multicolumn{3}{|c|}{ Post 2 vs. Post 3} \\
\hline & & & & $p$-value & $\Delta( \pm 90 \% \mathrm{CI})$ & $\mathrm{ES}( \pm 90 \% \mathrm{CI})$ & $p$-value & $\Delta( \pm 90 \% \mathrm{CI}$ & $\overline{\mathrm{ES}( \pm 90 \% \mathrm{CI})}$ & $p$-value & $\Delta( \pm 90 \% \mathrm{CI}$ & $\mathrm{ES}( \pm 90 \% \mathrm{CI})$ \\
\hline $\mathrm{T} 10(\mathrm{~s})$ & $1.87 \pm 0.09$ & $1.81 \pm 0.09$ & $1.87 \pm 0.06$ & 0.013 & $-3.6 \pm 1.7$ & $0.63 \pm 0.31$ & 1.000 & $0.1 \pm 2.1$ & $-0.01 \pm 0.37$ & 0.035 & $3.8 \pm 2.2$ & $-0.65 \pm 0.37$ \\
\hline $\mathrm{T} 20(\mathrm{~s})$ & $3.12 \pm 0.12$ & $3.07 \pm 0.10$ & $3.14 \pm 0.07$ & 0.153 & $-1.6 \pm 1.3$ & $0.37 \pm 0.31$ & 0.906 & $0.9 \pm 1.4$ & $-0.20 \pm 0.33$ & 0.037 & $2.5 \pm 1.5$ & $-0.56 \pm 0.34$ \\
\hline $\mathrm{CMJ}(\mathrm{cm})$ & $34.7 \pm 3.0$ & $39.0 \pm 4.1$ & $36.9 \pm 4.5$ & 0.002 & $12.3 \pm 4.4$ & $1.27 \pm 0.43$ & 0.259 & $5.9 \pm 6.0$ & $0.63 \pm 0.62$ & 0.300 & $-5.7 \pm 5.5$ & $-0.64 \pm 0.64$ \\
\hline $1 \mathrm{RM}_{\mathrm{est}}(\mathrm{kg})$ & $85.3 \pm 17.3$ & $94.6 \pm 16.2$ & $90.4 \pm 17.2$ & 0.003 & $11.4 \pm 4.6$ & $0.47 \pm 0.18$ & 0.07 & & $0.26 \pm 0.44$ & 0.085 & $-4.7 \pm 3.5$ & $-0.21 \pm 0.16$ \\
\hline $\mathrm{VO}_{2} \max \left(\mathrm{ml} \cdot \mathrm{kg}^{-1} \cdot \mathrm{min}^{-1}\right)$ & $43.6 \pm 4.4$ & $48.9 \pm 4.5$ & $44.5 \pm 6.0$ & 0.000 & $12.2 \pm 2.8$ & $1.00 \pm 0.22$ & 1.000 & $1.7 \pm 5.2$ & $0.15 \pm 0.45$ & 0.011 & $-9.4 \pm 4.4$ & $-0.86 \pm 0.42$ \\
\hline
\end{tabular}

Data are mean \pm SD

HLG: High-load group; Pre: initial evaluation; Post 1: Evaluation after training period; Post 2: evaluation after detraining period; $\Delta$ : percentage of change; ES: intragroup

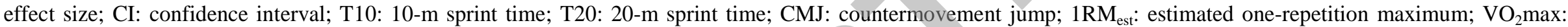
estimated maximal oxygen uptake. 
Table 6. Changes in selected neuromuscular performance variables from initial evaluation (pre) to final evaluation (post) between groups.

\begin{tabular}{|c|c|c|c|c|}
\hline & \multicolumn{4}{|c|}{ Changes observed for post- vs. pre } \\
\hline & \multirow[t]{2}{*}{$\begin{array}{l}\mathrm{P} \text { value between } \\
\text { groups }\end{array}$} & \multirow[t]{2}{*}{$\begin{array}{l}\text { Standarized differences } \\
\text { (Cohen: } 90 \% \mathrm{CI})\end{array}$} & \multicolumn{2}{|c|}{$\begin{array}{l}\text { Percent changes of better/trivial/ } \\
\text { worse effect }\end{array}$} \\
\hline \multicolumn{3}{|c|}{ (1) } & & \\
\hline LLG vs. CG & 0.148 & $0.38(0.13$ to 0.63$)$ & $89 / 11 / 0$ & Likely \\
\hline MLG vs. CG & 0.254 & $0.43(0.14$ to 0.71$)$ & $91 / 9 / 0$ & Likely \\
\hline HLG vs. CG & 0.000 & $0.80(0.45$ to 1.16$)$ & $99 / 1 / 0$ & Very Likely \\
\hline LLG vs. MLG & 1.000 & $0.05(-0.16$ to 0.25$)$ & $11 / 87 / 3$ & Likely Trivial \\
\hline LLG vs. HLG & 0.108 & $-0.40(-0.72$ to -0.07$)$ & $0 / 15 / 85$ & Likely harmful \\
\hline HLG vs. MLG & 0.057 & $0.52(0.15$ to 0.88$)$ & $93 / 7 / 0$ & Likely \\
\hline \multicolumn{5}{|l|}{ T20 } \\
\hline LLG vs. CG & 1.000 & $0.11(-0.06$ to 0.28$)$ & & Trivial \\
\hline MLG vs. CG & 0.436 & $0.29(0.08$ to 0.49$)$ & $3 / 0$ & Likely \\
\hline HLG vs. CG & 1.000 & $0.14(-0.14$ to 0.42$)$ & $35 / 62 / 3$ & Unclear \\
\hline LLG vs. MLG & 1.000 & $-0.19(-0.39$ to 0.02$)$ & $0 / 54 / 46$ & Possibly harmful \\
\hline LLG vs. HLG & 1.000 & $-0.03(-0.32$ to 0.27$)$ & $5 / 15$ & Unclear \\
\hline HLG vs. MLG & 1.000 & $-0.18(-0.54$ to 0.17$)$ & $5 / 49 / 46$ & Unclear \\
\hline \multicolumn{5}{|l|}{ CMJ } \\
\hline LLG vs. CG & 0.159 & $0.50(0.22$ to 0.78$)$ & $96 / 4 / 0$ & Very Likely \\
\hline MLG vs. CG & 0.031 & $0.67(0.28$ to 1.05$)$ & $97 / 3 / 0$ & Very Likely \\
\hline HLG vs. CG & 0.093 & $0.69(0.31$ to 1.08$)$ & $98 / 2 / 0$ & Very Likely \\
\hline LLG vs. MLG & 1.000 & $-0.13(-0.52$ to 0.26$)$ & $8 / 55 / 37$ & Unclear \\
\hline LLG vs. HLG & 1.000 & $-0.04(-0.41$ to 0.32$)$ & $13 / 64 / 23$ & Unclear \\
\hline HLG vs. MLG & 1.000 & $-0.12(-0.65$ to 0.41$)$ & $15 / 45 / 40$ & Unclear \\
\hline \multicolumn{5}{|l|}{$1 \mathbf{R M}_{\text {est }}$} \\
\hline LLG vs. CG & $0.00-7$ & 0.48 (0.30 to 0.66$)$ & $99 / 1 / 0$ & Very Likely \\
\hline MLG vs. CG & 0.043 & $0.36(0.20$ to 0.53$)$ & $95 / 5 / 0$ & Likely \\
\hline HLG vs. CG & 0.016 & $0.41(0.25$ to 0.58$)$ & $98 / 2 / 0$ & Very Likely \\
\hline LLG vs. MLG & 1.000 & $0.16(-0.11$ to 0.43$)$ & $39 / 59 / 2$ & Possibly \\
\hline LLG vs. HLG & 1.000 & $0.10(-0.17$ to 0.37$)$ & $26 / 71 / 3$ & Possibly \\
\hline HLG vs. MLG & 1.000 & $0.06(-0.19$ to 0.31$)$ & $17 / 79 / 4$ & Likely Trivial \\
\hline \multicolumn{5}{|l|}{$\mathrm{VO}_{2} \max$} \\
\hline LLG vs. CG & 0.004 & 0.54 (0.33 to 0.74$)$ & $99 / 1 / 0$ & Very Likely \\
\hline MLG vs. CG & 0.035 & $0.54(0.28$ to 0.80$)$ & $98 / 2 / 0$ & Very Likely \\
\hline HLG vs. CG & 0.037 & 0.73 (0.51 to 0.95$)$ & $100 / 0 / 0$ & Most Likely \\
\hline LLG vs. MLG & 1.000 & $0.13(-0.14$ to 0.39$)$ & $32 / 66 / 2$ & Possibly \\
\hline LLG vs. HLG & 1.000 & $0.14(-0.11$ to 0.39$)$ & $34 / 64 / 2$ & Possibly \\
\hline HLG vs. MLG & 1.000 & 0.00 (-0.31 to 0.32$)$ & $15 / 71 / 14$ & Unclear \\
\hline
\end{tabular}

CI: confidence interval; LLG: Low-load group; MLG: Moderate-load group; HLG: High-load group; CG: Control group; T10: 10-m sprint time; T20: 20-m sprint time; CMJ: countermovement jump; 1RM $\mathrm{est}_{\text {: }}$ estimated one-repetition maximum; $\mathrm{VO}_{2}$ max: estimated maximal oxygen uptake. Note: all differences are presented as improvements for the first group compared with the second group (i.e., LLG vs. CG), so that negative and positive differences are in the same direction. 
A

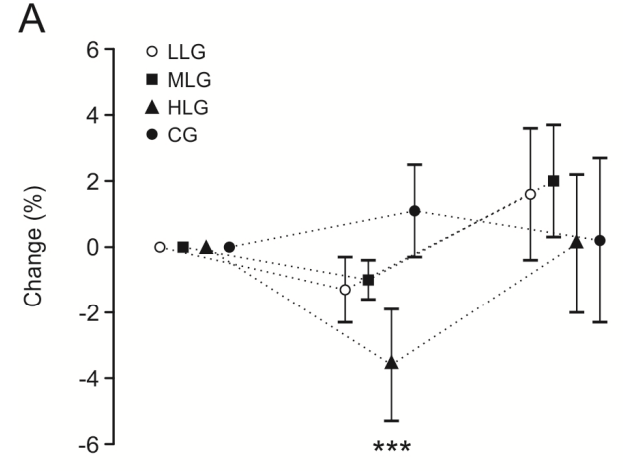

B

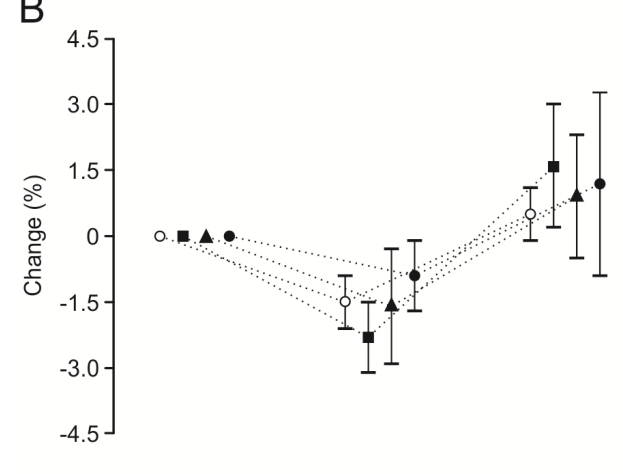

C

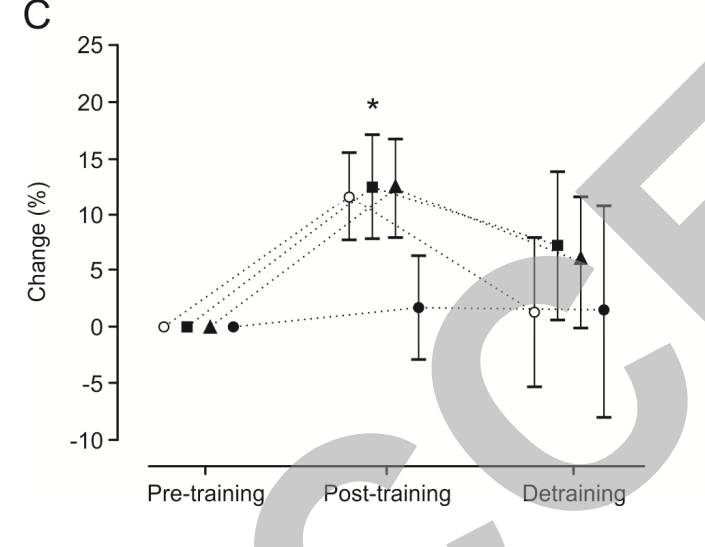

D

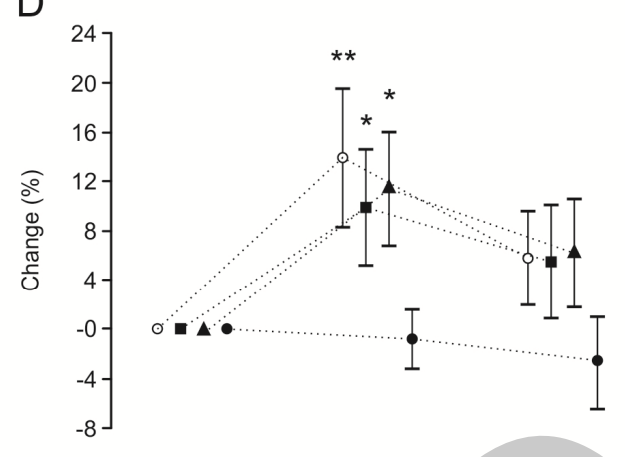

E

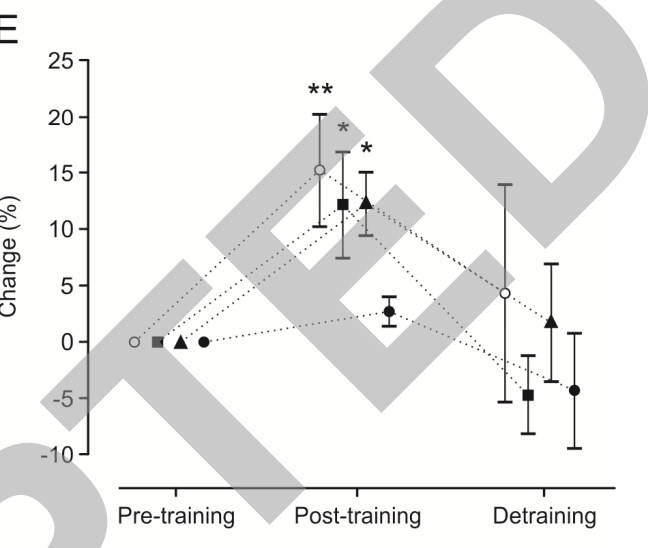

